\title{
THE TOPOLOGICAL CONGRESS IN MOSCOW
}

The first International Topological Conference met in Moscow, September 4-10, 1935, at the call of the Mathematical Institute of the University of Moscow. There were thirty-seven delegates, twelve from the Soviet Union and twenty-five from nine foreign countries, including ten from America; there were also about twenty visitors in attendance, mostly from parts of the USSR. The group was noteworthy for its youthfulness and vigor; in its small number it included a large proportion of the active topologists of the world. The names of the Americans present are given on page 615 of the September issue of this Bulletin. The Conference devoted all available time, except one day reserved for excursions, to papers and discussion. The papers showed a broad conception of topology and its relations with other subjects. The discussions which followed the papers were keen and informal; they revealed many instances of overlapping investigations, and led to an invaluable exchange of ideas. In fact one could hardly conceive of a more successful scientific gathering. The reception accorded the foreign delegates by the mathematicians and officials of the USSR was most cordial, and the hospitality most generous.

K. Borsuk, E. Čech, H. Freudenthal, I. I. Gordon, and S. Lefschetz presented papers on various phases of Homology theory; J. Nielsen, P. A. Smith, and A. Tychonoff on Transformations; J. W. Alexander, A. Kolmogoroff, and A. W. Tucker on Abstract topology; S. Cohn-Vossen, H. Hopf, and H. Whitney on Differential manifolds; N. K. Bruschlinsky, V. A. Efremovitch, and J. Rozanska on Continuous representations; C. Kuratowski, S. Mazurkiewicz, and W. Sierpinski on Point-set theory; Garrett Birkhoff, E. R. van Kampen, J. von Neumann, L. Pontrjagin, and A. Weil on Continuous groups; D. van Dantzig and M. H. Stone on Topological algebra; N. Bogoliuboff and V. W. Niemytzki on Topological mechanics; P. Heegaard on the Four-color problem; G. de Rham on Lens-spaces; A. A. Markoff on Tresses; O. Zariski on Algebraic geometry; J. Schauder on Function-space; W. Hurewicz on Homotopy; G. Nöbeling on Triangulation; and P. Alexandroff (president of the Conference) on Dimension theory and other topics.

A. W. TUCKeR 